\title{
DIELECTRONIC RECOMBINATION IN THE GASEOUS NEBULAE AS A COOLING PROCESS
}

\author{
A.F. KHOLTYGIN
}

S.-Petersbourg Univ. Astron. Obs., 198904, St.Petergof, Russia

The dielectronic recombination (DR) is of importance at 'low' (nebular) temperature [1]. This process leads to cooling the electron gas in nebulae. The cooling rate by recombination of ion $X^{+n}$ is

$$
L_{d r}\left(T_{e}\right)=\sum_{j} \frac{4 \pi^{3 / 2} a_{0}^{3}}{\left(k T_{e} / R y\right)^{3 / 2}} \frac{g_{j} W_{j}^{a}}{g^{+}} \exp \left(-\frac{\Delta E_{j}}{k T_{e}}\right) \frac{W_{j}^{r}}{W_{j}^{a}+W_{j}^{r}} \Delta E_{j} .
$$

Here $W_{j}^{r}$ and $W_{j}^{a}$ are respectively the radiation and autoionization probabilities for the autoionization state $j$ of ion $X^{+n}, \Delta E_{j}$ is the energy of this state, $g_{j}$ and $g^{+}$ are respectively the statistical weights of the state $j$ and the ground state of the ion $X^{+n+1}$. We have calculated $L_{d r}\left(T_{e}\right)$ for all ions of C. It is shown that the process of DR cooling is important only for nebulae with extraordinarily high abundances $\left(\left\{C^{+i} / H^{+}\right\}>0.01\right)$ of these ions.

\section{References}

[1]. Nussbaumer H., Storey P.J. (1984) 'Dielectronic recombination at low temperature.II: Recombination coefficients for lines at C, N, O' , Astronomy and Astrophysics, $\underline{56}, 293-312$. 